Supporting Information

\title{
Gold Nanoparticle Thin Film-Based Strain Sensors for Monitoring Human Pulse
}

Wei-Wun Jheng ${ }^{1}, Y u$-Shun Su², Yun-Lien Hsieh ${ }^{1}$,Yu-Jhan Lin ${ }^{3}$, Shien-Der Tzeng ${ }^{4}$, Chih-Wei Chang ${ }^{3}$, Jenn-Ming Song ${ }^{2}$ and Watson Kuo ${ }^{1 *}$

${ }^{1}$ Department of Physics, National Chung Hsing University, Taichung 402, Taiwan

${ }^{2}$ Department of Materials Science and Engineering, National Chung Hsing University, Taichung 402, Taiwan

${ }^{3}$ Center for Condensed Matter Sciences, National Taiwan University, Taipei 106, Taiwan

${ }^{4}$ Department of Physics, National Dong Hwa University, Hualien 974, Taiwan

\section{Corresponding Author}

*email:wkuo@phys.nchu.edu.tw 


\section{Device packaging}

To package our sensors, we prepare two identical PDMS strips deposited with Au nanoparticle (NP) film. As illustrated in Fig. S1, parts of the PDMS strip( $\sim 5 \mathrm{~mm}$ long) were cleaned with alcohol for removing deposited AuNPs. The cleaned areas were subsequently coated by silver paste, followed by placement of two thin copper wires to form measurement electrodes. Then the strip was bonded with another flipped strip with cleaned areas.

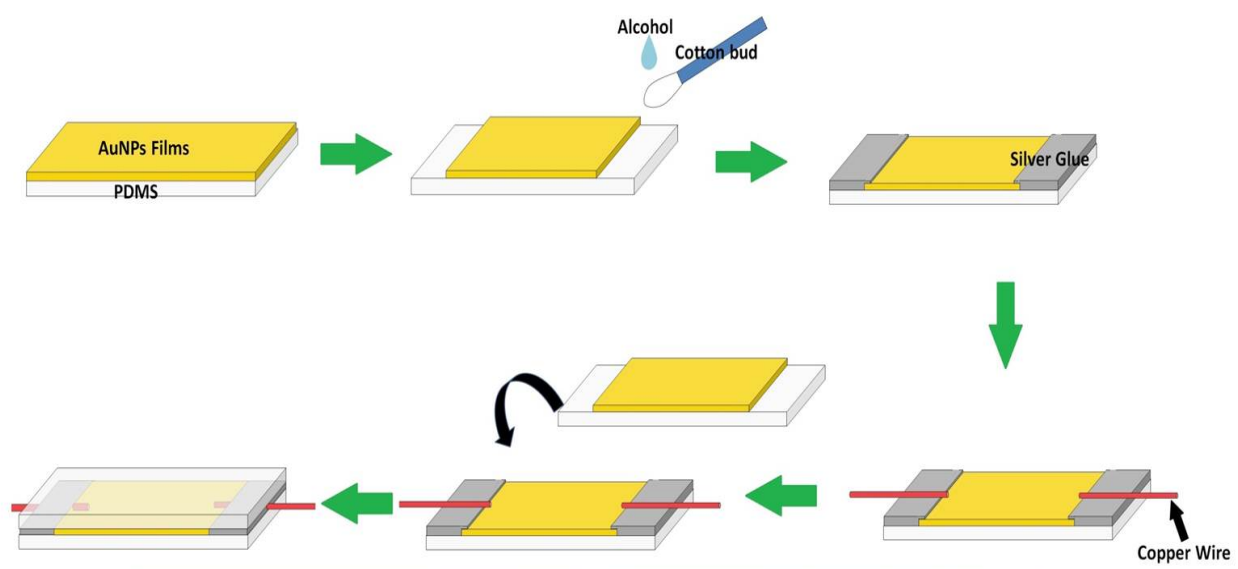

Fig S1. The procedures for device packaging. 


\section{Frequency response}

As illustrated in Fig. S2, two methods were applied to introduce vibrations to the device: fully attachment and single pint contact. The former is done by directly attach the device to the speaker diaphragm. Several positions were chosen to yield similar results. For the later, the loud speaker was arranged upside down while the device was suspended on a micro positioning stage. One can adjust the device position in such a way that the diaphragm made a single point contact at the center of device.

For calibrating the driving loud speaker, we perform the measurement of sound pressure level(SPL) of the speaker in an anechoic chamber as shown in Fig. S3(a). Fig. S3(b) and (c) respectively illustrate the SPL data and converted displacement under a constant voltage driving. For frequency between 10 and $100 \mathrm{~Hz}$, we made an assumption $s \sim f^{0.5}$, in which $s$ is the displacement and $f$ is the frequency. The calibration is done by assuming the device resistance change is proportional to the displacement. 

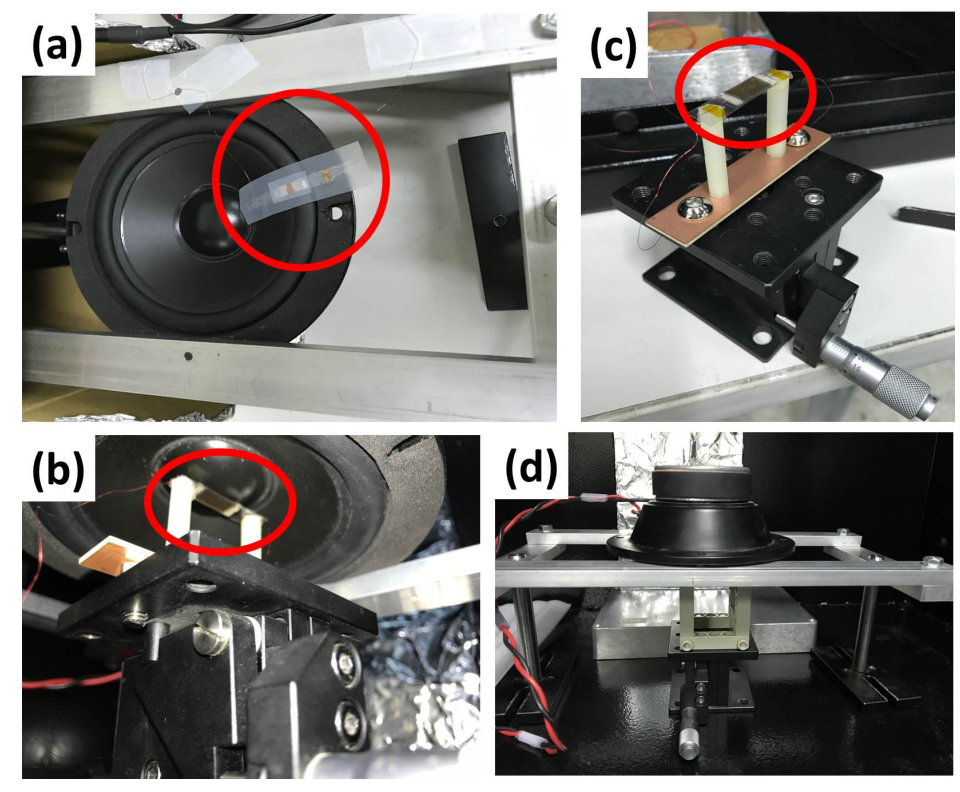

Fig. S2 Two methods were applied to introduce vibrations to the device. (a)Fully attachment method. (b)(c)(d) Single point contact.

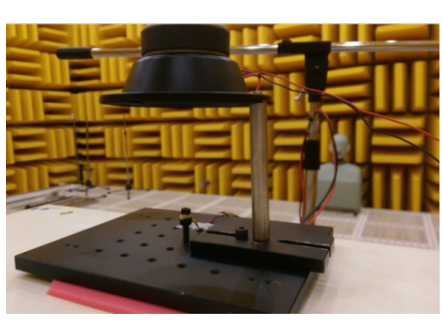

(a)
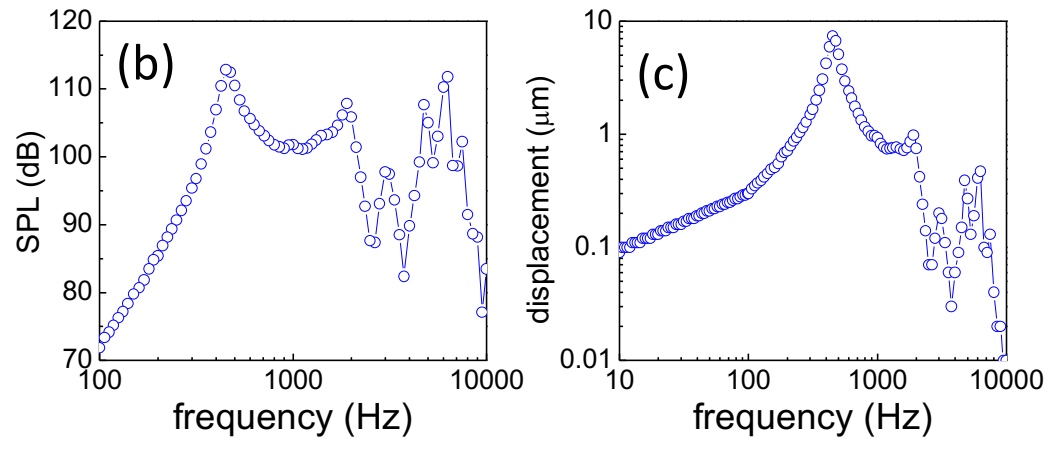

Fig. S3 (a) the driving speaker is under test in an anechoic chamber. (b) The sound pressure level(SPL) determined in the test. (c) the displacement calculated from the SPL. 


\section{NP layer dependence of gauge factor}

The devices made of two sizes of NPs regardless deposition layers may show different distributions in their gauge factor as illustrated in Fig. S4 (a), agreeing that a larger NP size would yield a greater gauge factor. When the particle size is fixed, an effected way to reduce the resistance is to increase the deposited NP layer. On average, a device with more NP layers has a lower resistance. How the device resistance and layer affect the gauge factor can be illustrated in Fig. S4(b) and (c) respectively. The plots contain sample sets of 4 layers of $12 \mathrm{~nm} \mathrm{NPs} \mathrm{and} \mathrm{8,} \mathrm{11,}$ 14 and 17 layers of $16 \mathrm{~nm}$ NPs. A general result is the intermediate resistance, roughly 3-6 M $\Omega$ displays the best gauge factor. However, the total layer number of the thin film does not have a clear impact to the result.
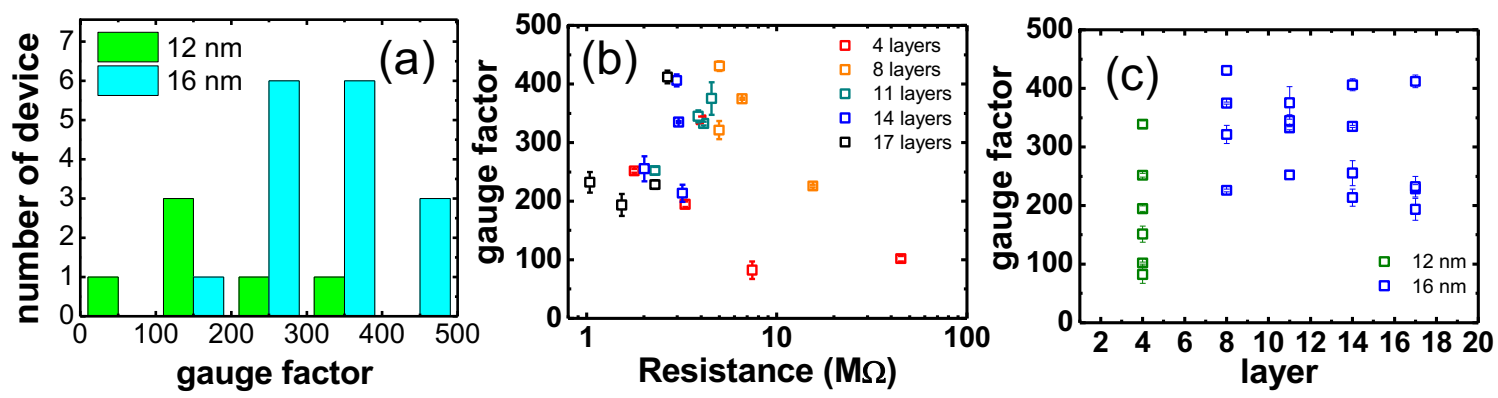

Fig. S4 (a) The histogram of gauge factors of PDMS devices made of $12 \mathrm{~nm}$ (4 layers) and $16 \mathrm{~nm}$ NPs(8-17 layers). (b) The relation between gauge factor and as-fabricated resistance. (c) The gauge factor as a function of NP layers. 


\section{Comparison of polyimide and PDMS substrates}

The same AuNPs were deposited on to PDMS substrates and polyimide (PI) substrates using the same centrifugal method, but yield very different performance for the two cases. As illustrated in Fig. S5, the histogram of 22 PDMS devices under study demonstrated much higher gauge factor than that of PI devices. Therefore, the working principle for PDMS devices may deviate the believed ideal model of uniformly separated AuNPs under strain. Instead, it is believed that percolation can better explain the observed phenomenon in PDMS case.

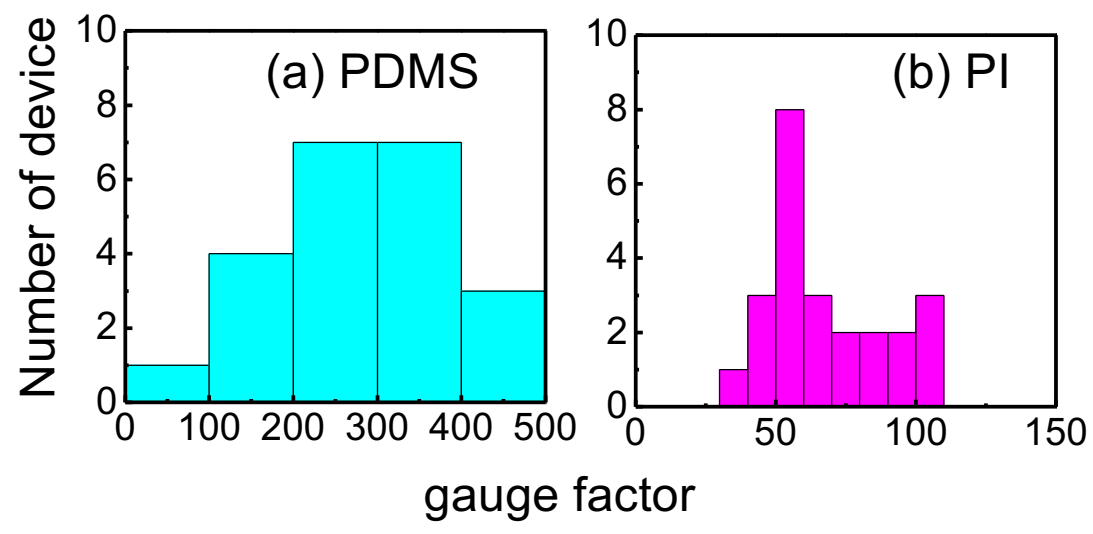

Fig. S5 (a)(b) The histograms of gauge factor of devices made on PDMS substrates(a) and polyimide (PI) substrates(b). The gauge factors of PDMS devices are much greater than that of PI ones. 


\section{Resistance change and Faraday induction}

In the frequency response measurement, we found that the monitored modulated device voltage at a fixed bias current was not only contributed from resistance change, but also Faraday induction. To clarify the origin, we may compare the measurement results for un-biased and biased conditions. In general, resistance change has a linear bias dependence, but induction appears the same in any bias conditions. As illustrated in Fig. S6, at rather low frequencies, the voltage modulations at zero-bias is negligible, but it grows as frequency increases. Because of the biased and un-biased voltage modulations are almost the same for $2 \mathrm{kHz}$, the voltage modulation mostly contributed from induction rather than resistance change.
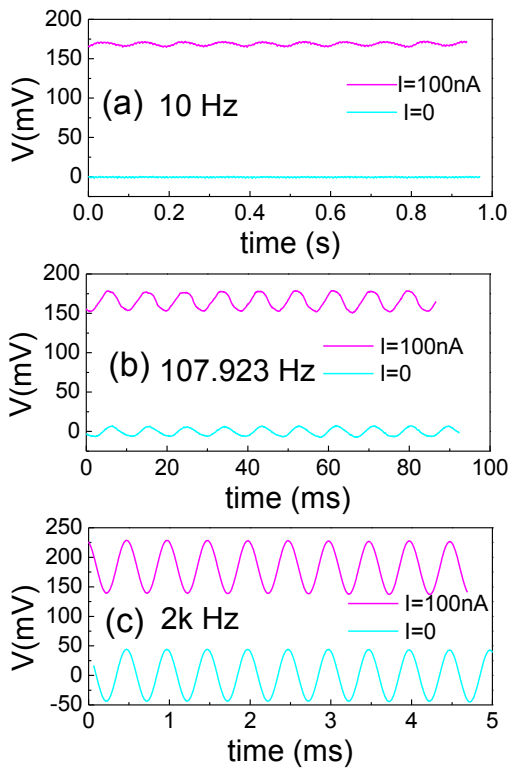

Fig S6. The real-time scans of voltage when the device was under zero-biased and under a 100nA bias current at speaker driving frequencies of $10 \mathrm{~Hz}(\mathrm{a}), 107.923 \mathrm{~Hz}(\mathrm{~b})$, and 2k Hz(c). It clear shows that when the frequency increases, a sinusoidal change of voltage at zero bias is getting greater due to Faraday induction. 